\title{
Rotational spectroscopic study and astronomical search for propiolamide in Sgr B2(N) ${ }^{\star}$
}

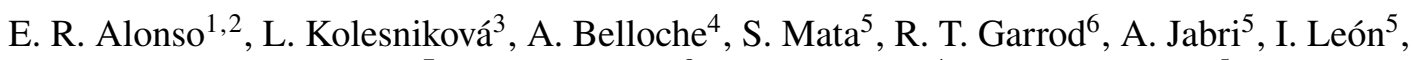 \\ J.-C. Guillemin ${ }^{7}$, H. S. P. Müller ${ }^{8}$, K. M. Menten ${ }^{4}$, and J. L. Alonso ${ }^{5}$ \\ ${ }^{1}$ Instituto Biofisika (UPV/EHU, CSIC), University of the Basque Country, Leioa, Spain \\ ${ }^{2}$ Fundación Biofísica Bizkaia / Biofisika Bizkaia Fundazioa (FBB), Barrio Sarriena s/n, Leioa, Spain \\ ${ }^{3}$ Department of Analytical Chemistry, University of Chemistry and Technology, Technická 5, 16628 Prague 6, Czech Republic \\ e-mail: Lucie.Kolesnikova@vscht.cz \\ ${ }^{4}$ Max-Planck-Institut für Radioastronomie, Auf dem Hügel 69, 53121 Bonn, Germany \\ ${ }^{5}$ Grupo de Espectroscopia Molecular (GEM), Edificio Quifima, Área de Química-Física, Laboratorios de Espectroscopia y \\ Bioespectroscopia, Parque Científico UVa, Unidad Asociada CSIC, Universidad de Valladolid, 47011 Valladolid, Spain \\ ${ }^{6}$ Departments of Chemistry and Astronomy, University of Virginia, Charlottesville, VA 22904, USA \\ ${ }^{7}$ Univ Rennes, Ecole Nationale Supérieure de Chimie de Rennes, CNRS, ISCR - UMR 6226, 35000 Rennes, France \\ ${ }^{8}$ I. Physikalisches Institut, Universität zu Köln, Zülpicher Str. 77, 50937 Köln, Germany
}

Received 22 December 2020 / Accepted 22 January 2021

\begin{abstract}
Context. For all the amides detected in the interstellar medium (ISM), the corresponding nitriles or isonitriles have also been detected in the ISM, some of which have relatively high abundances. Among the abundant nitriles for which the corresponding amide has not yet been detected is cyanoacetylene $(\mathrm{HCCCN})$, whose amide counterpart is propiolamide $\left(\mathrm{HCCC}(\mathrm{O}) \mathrm{NH}_{2}\right)$.

Aims. With the aim of supporting searches for this amide in the ISM, we provide a complete rotational study of propiolamide from 6 to $440 \mathrm{GHz}$.

Methods. Time-domain Fourier transform microwave spectroscopy under supersonic expansion conditions between 6 and $18 \mathrm{GHz}$ was used to accurately measure and analyze ground-state rotational transitions with resolved hyperfine structure arising from nuclear quadrupole coupling interactions of the ${ }^{14} \mathrm{~N}$ nucleus. We combined this technique with the frequency-domain room-temperature millimeter wave and submillimeter wave spectroscopies from 75 to $440 \mathrm{GHz}$ in order to record and assign the rotational spectra in the ground state and in the low-lying excited vibrational states. We used the ReMoCA spectral line survey performed with the Atacama Large Millimeter/submillimeter Array toward the star-forming region Sgr B2(N) to search for propiolamide.

Results. We identified and measured more than 5500 distinct frequency lines of propiolamide in the laboratory. These lines were fitted using an effective semi-rigid rotor Hamiltonian with nuclear quadrupole coupling interactions taken into consideration. We obtained accurate sets of spectroscopic parameters for the ground state and the three low-lying excited vibrational states. We report the nondetection of propiolamide toward the hot cores Sgr B2(N1S) and Sgr B2(N2). We find that propiolamide is at least 50 and 13 times less abundant than acetamide in Sgr B2(N1S) and Sgr B2(N2), respectively, indicating that the abundance difference between both amides is more pronounced by at least a factor of 8 and 2, respectively, than for their corresponding nitriles.

Conclusions. Although propiolamide has yet to be included in astrochemical modeling networks, the observed upper limit to the ratio of propiolamide to acetamide seems consistent with the ratios of related species as determined from past simulations. The comprehensive spectroscopic data presented in this paper will aid future astronomical searches.
\end{abstract}

Key words. ISM: molecules - astrochemistry - line: identification - ISM: individual objects: Sagittarius B2 astronomical databases: miscellaneous

\section{Introduction}

The discovery of molecules in the interstellar medium (ISM) is greatly facilitated by our ability to predict which compounds are present in that environment. Many interstellar molecules form in the icy mantles of dust grains, including complex organic molecules (COMs ${ }^{1}$; Herbst \& van Dishoeck 2009). Laboratory simulations of the chemistry of grains in the ISM could provide lists of target compounds. For example, it has recently

* Full Table 2 is only available at the CDS via anonymous ftp to cdsarc.u-strasbg.fr (130.79.128.5) or via http://cdsarc. u-strasbg.fr/viz-bin/cat/J/A+A/647/A55

1 COMs are defined to contain six or more atoms; at least one of these atoms is carbon. been shown that, under hydrogen radical bombardments at $10 \mathrm{~K}$, the aldehydes are not reduced into the corresponding alcohols (Jonusas et al. 2017) except for formaldehyde which gives methanol (Hiraoka et al. 1994; Watanabe \& Kouchi 2002; Fuchs et al. 2009; Pirim \& Krim 2011). Based on similar experiments, nitriles do not lead to amines (Krim et al. 2019) except for HCN, which provides methylamine in a very low yield (Theule et al. 2011). On the other hand, to the best of our knowledge, the relation between nitriles and amides by the hydration of the former or the dehydration of the latter (see Fig. 1) has never been studied in such laboratory simulations. We can however observe that some couples of nitrile and amide are present in the list of compounds detected in the ISM, such as hydrogen cyanide $(\mathrm{HCN})$ and formamide $\left(\mathrm{NH}_{2} \mathrm{CHO}\right)$ or acetonitrile $\left(\mathrm{CH}_{3} \mathrm{CN}\right)$ and 


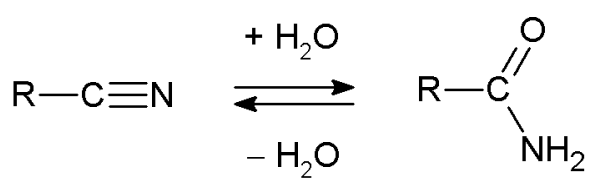

Fig. 1. Schematic representation of the hydration of nitriles to amides and the dehydration of amides to nitriles.

acetamide $\left(\mathrm{CH}_{3} \mathrm{C}(\mathrm{O}) \mathrm{NH}_{2}\right)$. However, such comparisons are difficult because for most of the amides corresponding to the nitriles observed in the ISM, one of the most powerful tools for such detections, the millimeter wave spectrum, has never been recorded. Only microwave spectra can be found for propionamide (Marstokk et al. 1996), acrylamide (Marstokk et al. 2000), or propiolamide (Little \& Gerry 1978). The particular case of glycinamide $\left(\mathrm{NH}_{2} \mathrm{CH}_{2} \mathrm{C}(\mathrm{O}) \mathrm{NH}_{2}\right)$ has to be detailed. Many attempts have tried to detect amino acids in the ISM and particularly the simplest one, glycine, but only a potential precursor of glycine, aminoacetonitrile $\left(\mathrm{NH}_{2} \mathrm{CH}_{2} \mathrm{CN}\right)$, has been detected (Belloche et al. 2008). In the hypothesis that this compound could be a precursor of glycine by hydrolysis, the addition of one molecule of water would give the glycinamide intermediate. The microwave spectrum of this compound was recently published (Alonso et al. 2018), but the millimeter wave spectrum is still missing. On the other hand, this is not the case of glycolamide, a glycine isomer, whose millimeter wave spectrum was recorded very recently (Sanz-Novo et al. 2020).

To support the hypothesis of a correlation between the presence of a nitrile and its corresponding amide, we first looked at which nitriles are the most abundant in the ISM. Unambiguously, after hydrogen cyanide $(\mathrm{HCN})$ and acetonitrile $\left(\mathrm{CH}_{3} \mathrm{CN}\right)$, propionitrile $\left(\mathrm{C}_{2} \mathrm{H}_{5} \mathrm{CN}\right)$ and cyanoacetylene $\left(\mathrm{HC}_{3} \mathrm{~N}\right)$ are the most abundant derivatives (McGuire 2018). $\mathrm{HC}_{3} \mathrm{~N}$ is ubiquitous in the Universe, having been detected in comets, the atmosphere of Titan, and in many places of the ISM. Therefore, propiolamide $\left(\mathrm{HCCC}(\mathrm{O}) \mathrm{NH}_{2}\right)$ is a plausible interstellar candidate. In addition, this eight-atom molecule has fewer atoms than the detected acetamide and contains a carbon-carbon triple bond, a widely represented functional group among the known circumstellar and interstellar compounds such as cyanopolyynes, alkynes, alkyne radicals or anions, magnesium acetylide $(\mathrm{MgCCH})$, propynal (HCCCHO), ethynyl isocyanide ( $\mathrm{HCCNC})$, oxo-penta-2,4diynyl $\left(\mathrm{HC}_{5} \mathrm{O}\right)$, and oxo-hepta-2,4,6-triynyl $\left(\mathrm{HC}_{7} \mathrm{O}\right)(\mathrm{McGuire}$ 2018).

Since reliable interstellar searches for propiolamide should be based on rotational transitions measured directly in the laboratory or those predicted from a data set that covers a broad spectral range, in this work we provide a detailed rotational study of this amide up to $440 \mathrm{GHz}$. Two different high-resolution spectroscopic techniques were employed for this purpose. Narrowband cavity-based Fourier transform microwave (FTMW) spectroscopy was used to measure the jet-cooled spectrum of propiolamide up to $18 \mathrm{GHz}$ and to analyze the ${ }^{14} \mathrm{~N}$ nuclear quadrupole hyperfine structure of the ground-state rotational transitions. Millimeter and submillimeter wave spectroscopic techniques were used to record the room-temperature spectrum up to $440 \mathrm{GHz}$ and to identify higher- $J$ ground-state transitions and the pure rotational spectra in excited vibrational states. The present work significantly extends the knowledge of the rotational spectrum of propiolamide and provides sufficiently precise laboratory information to search for this amide in space. We chose as a target for this interstellar search the high-mass starforming region Sagittarius (Sgr) B2(N), which is located close to the Galactic center. We use our latest imaging spectral line survey performed toward this source with the Atacama Large Millimeter/submillimeter Array (ALMA) in the $3 \mathrm{~mm}$ atmospheric window, the ReMoCA survey, which recently led to the identification of urea (Belloche et al. 2019).

The article is structured as follows. Details about the laboratory experiments are given in Sect. 2 and the analysis of the recorded spectra of propiolamide is described in Sect. 3. The search for propiolamide toward Sgr B2(N) is reported in Sect. 4. We discuss our results in Sect. 5 and give our conclusions in Sect. 6.

\section{Experiments}

Propiolamide was prepared from methyl propiolate, following the recipe of Miller \& Lemmon (1967). The solid product was then evacuated overnight to remove traces of methanol and used without further purifications. To record the jet-cooled rotational spectrum in the $6-18 \mathrm{GHz}$ frequency range, propiolamide (melting point $57-62^{\circ} \mathrm{C}$ ) was heated to approximately $80^{\circ} \mathrm{C}$ in a pulsed nozzle, seeded in neon carrier gas (backing pressure of 1 bar) and adiabatically expanded into the Fabry-Pérot resonator of the FTMW spectrometer described elsewhere (Alonso et al. 2015; León et al. 2017). A short microwave radiation pulse of $0.3 \mathrm{~ms}$ duration was applied to polarize the molecules of propiolamide. A free induction decay associated with molecular emission was registered in the time domain and converted to the frequency domain by Fourier transformation. Since the molecular beam was directed along the resonator axis, the rotational transitions were observed as Doppler doublets. The resonance frequency was subsequently obtained as the arithmetic mean of the two Doppler components.

To record the room-temperature rotational spectra between 75 and $440 \mathrm{GHz}$, propiolamide was placed into a small glass container that was connected directly to the free space cell of the millimeter wave spectrometer and evacuated. The sample was heated with a heating gun (temperature of $80{ }^{\circ} \mathrm{C}$ of the heated air) until the pressure in the cell reached the optimal value between 10 and $20 \mu$ bar. The millimeter wave spectrometer employed in this work is based on sequential multiplication of the fundamental synthesizer frequency $(\leq 20 \mathrm{GHz})$ by a set of active and passive multipliers (VDI, Inc) and has been described elsewhere (Kolesniková et al. 2017a,b). For the measurements reported in this work, the synthesizer output was frequency modulated at a modulation frequency of $10.2 \mathrm{kHz}$ and modulation depth between 20 and $40 \mathrm{kHz}$ before it was multiplied by amplifier-multiplier chains (WR10.0, WR6.5, WR9.0) in combination with additional doublers (WR4.3, WR2.2) and a tripler (WR2.8). After a double pass of the radiation through the cell, the signal was detected by zero-bias detectors and demodulated by a lock-in amplifier tuned to twice the modulation frequency. This demodulation procedure results in a shape of the lines that approximates the second derivative of a Gaussian line-profile function. All spectra were registered in $1 \mathrm{GHz}$ sections in both directions (a single acquisition cycle) and averaged. These sections were ultimately combined into a single spectrum and further processed using the AABS package (Kisiel et al. 2005, 2012).

\section{Rotational spectra and analysis}

\subsection{Ground-state rotational spectrum}

As revealed by Stark modulation spectroscopy, propiolamide is essentially planar with dipole moment components 

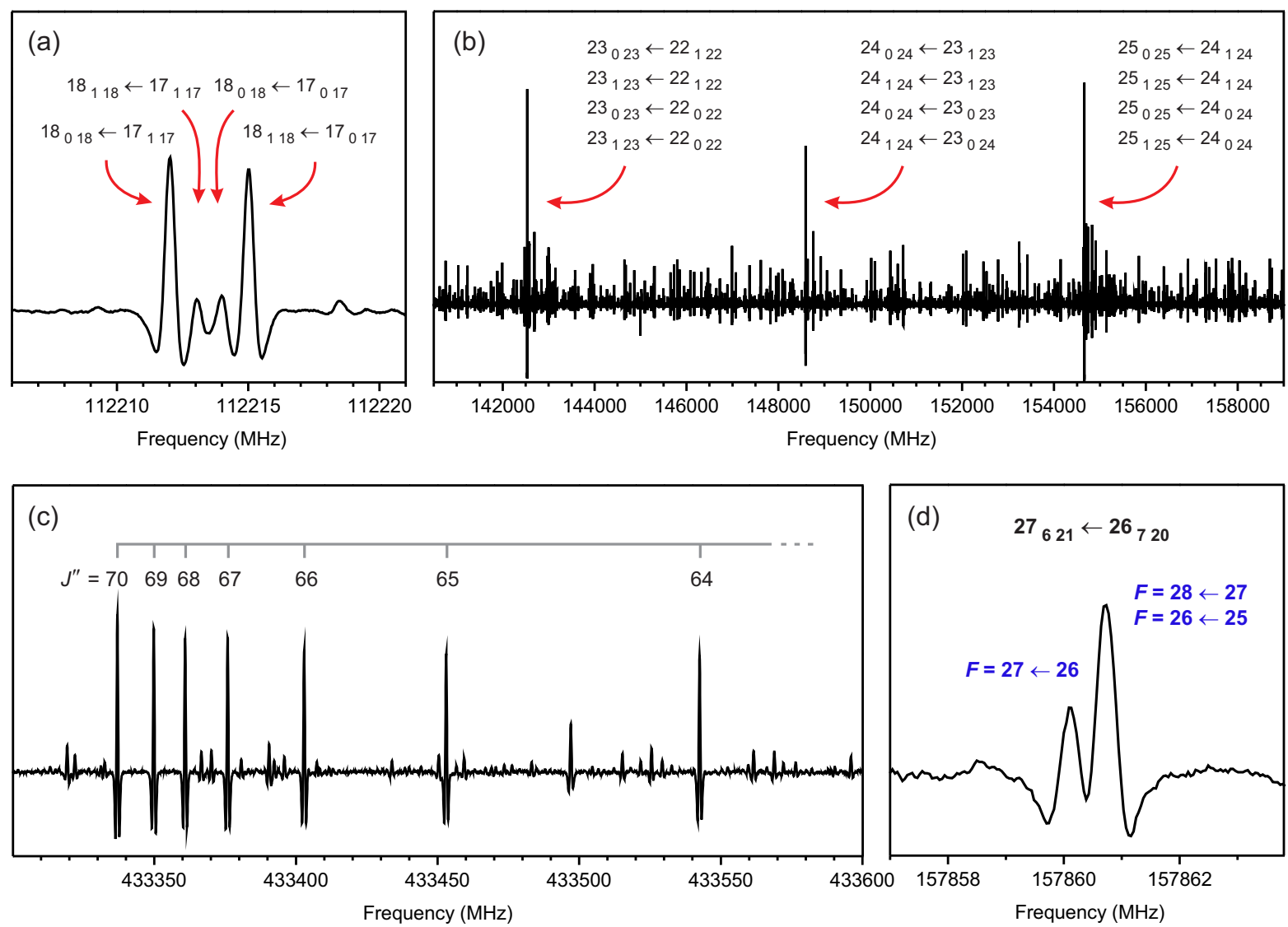

Fig. 2. Illustrations of the main characteristics of the room-temperature rotational spectrum of propiolamide. (a) An example of a quartet of $a$-type and $b$-type R-branch rotational transitions between $K_{a}=0,1, K_{c}=J$ pairs of energy levels. (b) Quadruply degenerate lines consisting of a pair of $a$-type and a pair of $b$-type transitions involving the same $K_{a}=0,1$ pairs of levels. These strong lines appear in the spectrum approximately every $6.06 \mathrm{GHz}$, which corresponds to the value of $2 C$. (c) An example of a group of high- $J$ quadruply degenerate transitions. The leading transition is $J=71 \leftarrow 70$ and the value of $J$ decreases by 1 with each successive line running to higher frequencies. The $K_{a}$ quantum number is increasing away from the leading line in such a way that the first line corresponds to four degenerate $K_{a}=0,1$ transitions, the second to $K_{a}=1,2$, the third to $K_{a}=2,3$, and so on. $(d)$ An example of ${ }^{14} \mathrm{~N}$ nuclear quadrupole hyperfine structure observed in the millimeter wave spectrum. The $F=J \pm 1$ hyperfine components typically appear overlapped, while the $F=J$ component is, in many cases, relatively well separated.

$\left|\mu_{a}\right|=1.08(2) \mathrm{D}$ and $\left|\mu_{b}\right|=3.51(2) \mathrm{D}$ (Little \& Gerry 1978). Both $a$-type and $b$-type transitions are therefore relevant in the millimeter wave spectrum. Strong lines corresponding to pairs of $b$-type transitions $(J+1)_{1, J+1} \leftarrow J_{0, J}$ and $(J+1)_{0, J+1} \leftarrow J_{1, J}$ could be immediately assigned in the spectrum. They were accompanied by weaker pairs of $a$-type transitions $(J+1)_{0, J+1} \leftarrow$ $J_{0, J}$ and $(J+1)_{1, J+1} \leftarrow J_{1, J}$. Figure 2 a shows that the two $b$ type transitions straddle the pair of $a$-type transitions giving rise to characteristic quartets. These quartets, however, are not observed throughout the whole spectrum. As the $J$ quantum number increases, the participating energy levels become near degenerate and the four members of the quartets coalesce into very prominent quadruply degenerate lines that clearly dominate the spectrum shown in Fig. 2b. The same quartet structures and line blending are also observed for higher $K_{a}$ transitions. With the $J$ quantum number further progressively increasing, the quadruply degenerate lines with low values of $K_{a}$ quantum numbers start to form groups in which successive lines differ by a unit in $J$ and $K_{a}$. A typical example of this feature is shown in Fig. 2c. Such a pattern is characteristic for high- $J a$-type and $b$-type spectra of planar and nearly planar molecules and was observed, for example, in acrylic acid (Alonso et al. 2015), phenol (Kolesniková et al. 2013), or 2-chloroacrylonitrile (Kisiel \& Pszczolkowski 1994). Finally, we also localized and measured b-type Q-branch transitions.

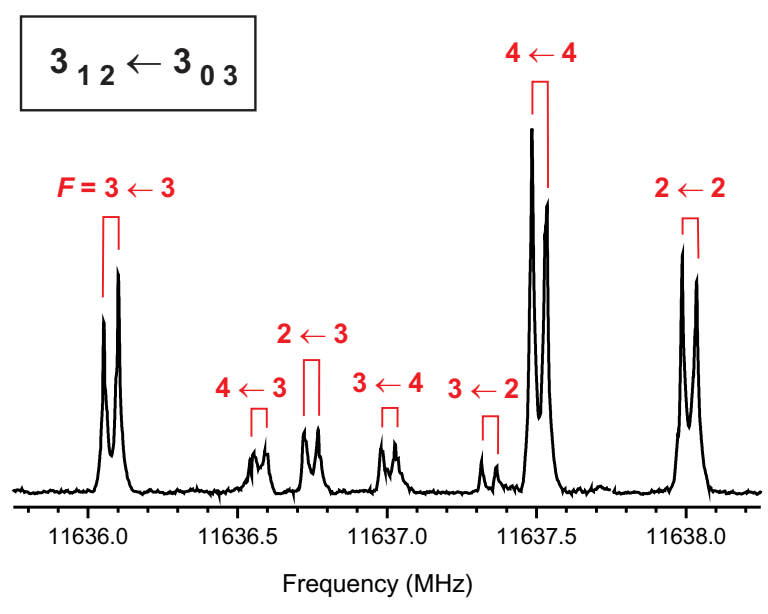

Fig. 3. ${ }^{14} \mathrm{~N}$ nuclear quadrupole hyperfine structure of the $3_{12} \leftarrow 3_{03}$ rotational transition as measured by cavity-based FTMW spectrometer. Each hyperfine component, which appears as a Doppler doublet, is labeled with the corresponding values of $F$ quantum number.

During the analysis procedure, we noticed that several transitions exhibited small splitting. We attributed this splitting to nuclear quadrupole coupling interactions of the single ${ }^{14} \mathrm{~N}$ nucleus present in the amide group. At the current resolution of 
Table 1. Spectroscopic constants of propiolamide in the ground state and three excited vibrational states ( $A$-reduction, $\mathrm{I}^{\mathrm{r}}$-representation).

\begin{tabular}{lrrrr}
\hline \hline & Ground state & $v_{\text {in }}=1$ & $v_{\text {out }}=1$ & $v_{\text {inv }}=1$ \\
\hline$A / \mathrm{MHz}$ & $11417.92091(11)^{(a)}$ & $11306.86167(25)$ & $11537.98892(27)$ & $11397.27733(18)$ \\
$B / \mathrm{MHz}$ & $4135.477101(40)$ & $4153.949250(78)$ & $4146.28849(10)$ & $4131.104876(83)$ \\
$C / \mathrm{MHz}$ & $3032.592907(35)$ & $3036.029785(61)$ & $3041.801213(72)$ & $3032.360188(56)$ \\
$\Delta_{J} / \mathrm{kHz}$ & $0.579701(18)$ & $0.612639(36)$ & $0.594171(59)$ & $0.578258(51)$ \\
$\Delta_{J K} / \mathrm{kHz}$ & $20.86142(18)$ & $19.27979(36)$ & $21.82228(41)$ & $20.73217(36)$ \\
$\Delta_{K} / \mathrm{kHz}$ & $-10.05819(20)$ & $-16.51167(89)$ & $-2.7538(10)$ & $-9.93128(41)$ \\
$\delta_{J} / \mathrm{kHz}$ & $0.1753084(80)$ & $0.190158(18)$ & $0.177915(29)$ & $0.174458(25)$ \\
$\delta_{K} / \mathrm{kHz}$ & $12.16107(24)$ & $11.28587(43)$ & $13.08620(65)$ & $12.08963(55)$ \\
$\Phi_{J} / \mathrm{Hz}$ & $0.0003369(31)$ & $0.0005094(77)$ & $0.000273(17)$ & $0.000370(15)$ \\
$\Phi_{J K} / \mathrm{Hz}$ & $0.19762(12)$ & $0.16406(25)$ & $0.22129(38)$ & $0.19630(28)$ \\
$\Phi_{K J} / \mathrm{Hz}$ & $-0.47001(41)$ & $-0.55885(90)$ & $-0.3560(13)$ & $-0.46592(78)$ \\
$\Phi_{K} / \mathrm{Hz}$ & $0.30222(35)$ & $-0.5968(10)$ & $1.1713(13)$ & $0.30279(69)$ \\
$\phi_{J} / \mathrm{Hz}$ & $0.0001448(15)$ & $0.0002278(41)$ & $0.0001229(89)$ & $0.0001680(78)$ \\
$\phi_{J K} / \mathrm{Hz}$ & $0.095653(64)$ & $0.08057(11)$ & $0.10591(22)$ & $0.09532(19)$ \\
$\phi_{K} / \mathrm{Hz}$ & $0.6385(11)$ & $0.3538(18)$ & $0.9125(29)$ & $0.6349(22)$ \\
$L_{J J K} / \mathrm{mHz}$ & $-0.0011188(88)$ & $-0.001301(27)$ & $-0.000844(35)$ & $-0.001004(28)$ \\
$L_{K K J} / \mathrm{mHz}$ & $\ldots$ & $0.05208(54)$ & $-0.05083(72)$ & $\ldots$ \\
$\chi_{a a} / \mathrm{MHz}$ & $1.8157(22)$ & $1.8157(b)$ & $1.8157(b)$ & $1.8157(b)$ \\
$\chi_{b b}-\chi_{c c} / \mathrm{MHz}$ & $6.0012(44)$ & $6.336(40)$ & $6.212(48)$ & $5.796(44)$ \\
$J_{\min } / J_{\max }$ & $0 / 75$ & $3 / 71$ & $3 / 71$ & $3 / 71$ \\
$K_{a, \min } / K_{a, \max }$ & $0 / 32$ & $0 / 25$ & $0 / 25$ & $0 / 25$ \\
$\sigma_{\text {fit }}(c) \mathrm{MHz}$ & 0.023 & 0.032 & 0.032 & 0.026 \\
$N^{(d)}$ & 1924 & 1482 & 1219 & 978 \\
\hline & & & &
\end{tabular}

Notes. ${ }^{(a)}$ The numbers in parentheses are $1 \sigma$ uncertainties (67\% confidence level) in units of the last decimal digit. The SPFIT/SPCAT program package (Pickett 1991) was used for the analysis. ${ }^{(b)}$ Fixed to the ground state value owing to the limited hyperfine data set in excited vibrational states. ${ }^{\left({ }^{()}\right.}$Root mean square deviation of the fit. ${ }^{(d)}$ Number of distinct frequency lines in the fit.

the spectra in Fig. 2, this typically gives rise to asymmetric doublets (see Fig. 2d). To correctly assign the hyperfine components blended together in these doublets, we measured and analyzed the nuclear quadrupole hyperfine structure from 6 to $18 \mathrm{GHz}$ using the cavity-based FTMW spectrometer that offers significantly higher spectral resolution. One of the measured transitions is exemplified in Fig. 3. In total 48 hyperfine components were measured and analyzed using the effective Hamiltonian $H=H_{\mathrm{R}}+H_{\mathrm{Q}}$, where $H_{\mathrm{R}}$ is the standard Watson semi-rigid rotor Hamiltonian in $A$-reduction and $\mathrm{I}^{\mathrm{r}}$ representation (Watson 1977), and $H_{\mathrm{Q}}$ represents the nuclear quadrupole coupling Hamiltonian (Gordy \& Cook 1984). The coupling scheme $F=J+I_{\mathrm{N}}$ between the rotational angular momentum $J$ and the ${ }^{14} \mathrm{~N}$ nuclear spin angular momentum $I_{\mathrm{N}}$ was used. A comparison of the observed asymmetric doublets with predictions based on this hyperfine analysis made it possible to undertake a simultaneous analysis of hyperfine-split and hyperfine-free lines. Transitions measured in the FTMW spectra and those in the millimeter wave spectra were adequately weighted assuming the experimental uncertainties of 5 and $30 \mathrm{kHz}$, respectively. These data were ultimately combined with a selection of hyperfine-free transitions from Little \& Gerry (1978) with assigned uncertainties equal to $100 \mathrm{kHz}$ and were analyzed using the same effective Hamiltonian as mentioned above. The broad coverage of transition types and quantum number values (see Table 1) guaranteed an accurate determination of rotational constants, centrifugal distortion constants along with the diagonal elements of the nuclear quadrupole coupling tensor. All these spectroscopic parameters are summarized in the first column of Table 1. The list of measured transitions is given in Table 2 of the supplementary material.

\subsection{Rotational spectra in excited vibrational states}

Each ground-state line in the millimeter wave spectrum was accompanied by satellite lines at the high- and low-frequency sides. In the neighborhood of the ground-state $J=17 \leftarrow 16$ transitions in Fig. 4, they appeared as quartets consisting of two $b$-type and two $a$-type transitions as in the ground state and were attributed to pure rotational transitions in excited vibrational states. According to our theoretical calculations at the B3LYP/6-311++G(d,p) level of the theory (Gaussian 16 package, Frisch et al. 2016) the three low-lying vibrational modes correspond mainly to the in-plane and out-of-plane bending motions of $\mathrm{C}-\mathrm{C} \equiv \mathrm{C}$ group $v_{\text {in }}\left(A^{\prime}\right)$ and $v_{\text {out }}\left(A^{\prime \prime}\right)$, respectively, and to the $\mathrm{NH}_{2}$ inversion $v_{\text {inv }}\left(A^{\prime \prime}\right)$. Using the calculated first-order vibration-rotation constants $\alpha_{i}$, it was possible to predict the rotational constants for relevant excited states according to the equation $B_{v}=B_{e}-\sum_{i} \alpha_{i}\left(v_{i}+1 / 2\right)$, where $B_{v}$ and $B_{e}$ denote all the three rotational constants in a given excited state and in equilibrium, respectively, and $v_{i}$ is the vibrational quantum number of the $i$-th mode. The predictions proved to be sufficiently accurate for the unambiguous assignment of the satellite pattern, as shown in Fig. 4. The two satellites displaced to high frequency from the ground state were assigned to the first quanta of the two bending modes $v_{\text {in }}=1$ and $v_{\text {out }}=1$, while the low-frequency satellite was ascribed to the first excited state of $\mathrm{NH}_{2}$ inversion $v_{\text {inv }}=1$ (see Fig. 4). Our assignments are in perfect agreement with those of Little \& Gerry (1978).

We performed the analysis of rotational transitions in the $v_{\text {in }}=1, v_{\text {out }}=1$, and $v_{\text {inv }}=1$ states in the same manner as for the ground state. The $a$-type and $b$-type R-branch transitions 
Table 2. Measured transitions in the ground state and three excited vibrational states of propiolamide.

\begin{tabular}{|c|c|c|c|c|c|c|c|c|c|c|c|c|}
\hline Vibrational state & $J^{\prime}$ & $K_{a}^{\prime}$ & $K_{c}^{\prime}$ & $F^{\prime}$ & $J^{\prime \prime}$ & $K_{a}^{\prime \prime}$ & $K_{c}^{\prime \prime}$ & $F^{\prime \prime}$ & $v_{\mathrm{obs}}(\mathrm{MHz})^{(a)}$ & $u_{\mathrm{obs}}(\mathrm{MHz})^{(b)}$ & $v_{\mathrm{obs}}-v_{\text {calc }}(\mathrm{MHz})^{(c)}$ & Comment ${ }^{(d)}$ \\
\hline G.S. & 2 & 0 & 2 & 1 & 1 & 1 & 1 & 1 & 6936.603 & 0.005 & -0.001 & (1) \\
\hline G.S. & 5 & 0 & 5 & $\ldots$ & 4 & 0 & 4 & $\ldots$ & 33827.730 & 0.100 & -0.036 & (2) \\
\hline G.S. & 28 & 5 & 23 & 28 & 28 & 4 & 24 & 28 & 109907.745 & 0.030 & -0.025 & (1) \\
\hline$v_{\text {in }}=1$ & 17 & 1 & 17 & $\ldots$ & 16 & 0 & 16 & $\ldots$ & 106268.500 & 0.030 & -0.015 & (1) \\
\hline$v_{\text {in }}=1$ & 24 & 6 & 18 & 24 & 23 & 7 & 17 & 23 & 114610.591 & 0.030 & -0.016 & (1) \\
\hline$v_{\text {out }}=1$ & 19 & 1 & 19 & $\ldots$ & 18 & 0 & 18 & $\ldots$ & 118650.237 & 0.030 & 0.015 & (1) \\
\hline$v_{\text {out }}=1$ & 25 & 3 & 22 & 25 & 25 & 2 & 23 & 25 & 123309.311 & 0.030 & 0.006 & (1) \\
\hline$v_{\text {inv }}=1$ & 16 & 2 & 15 & 16 & 16 & 1 & 16 & 16 & 89918.017 & 0.030 & 0.010 & (1) \\
\hline$v_{\text {inv }}=1$ & 10 & 5 & 6 & $\ldots$ & 9 & 4 & 5 & $\ldots$ & 141533.837 & 0.030 & 0.006 & (1) \\
\hline
\end{tabular}

Notes. ${ }^{(a)}$ Observed frequency. ${ }^{(b)}$ Uncertainty of the observed frequency. ${ }^{(c)}$ Observed minus calculated frequency. ${ }^{(d)}(1)$ This work. $(2)$ Taken from Little \& Gerry (1978). This table is available in its entirety in electronic form at the CDS. A portion is shown for guidance regarding its form and content.

\section{GS}

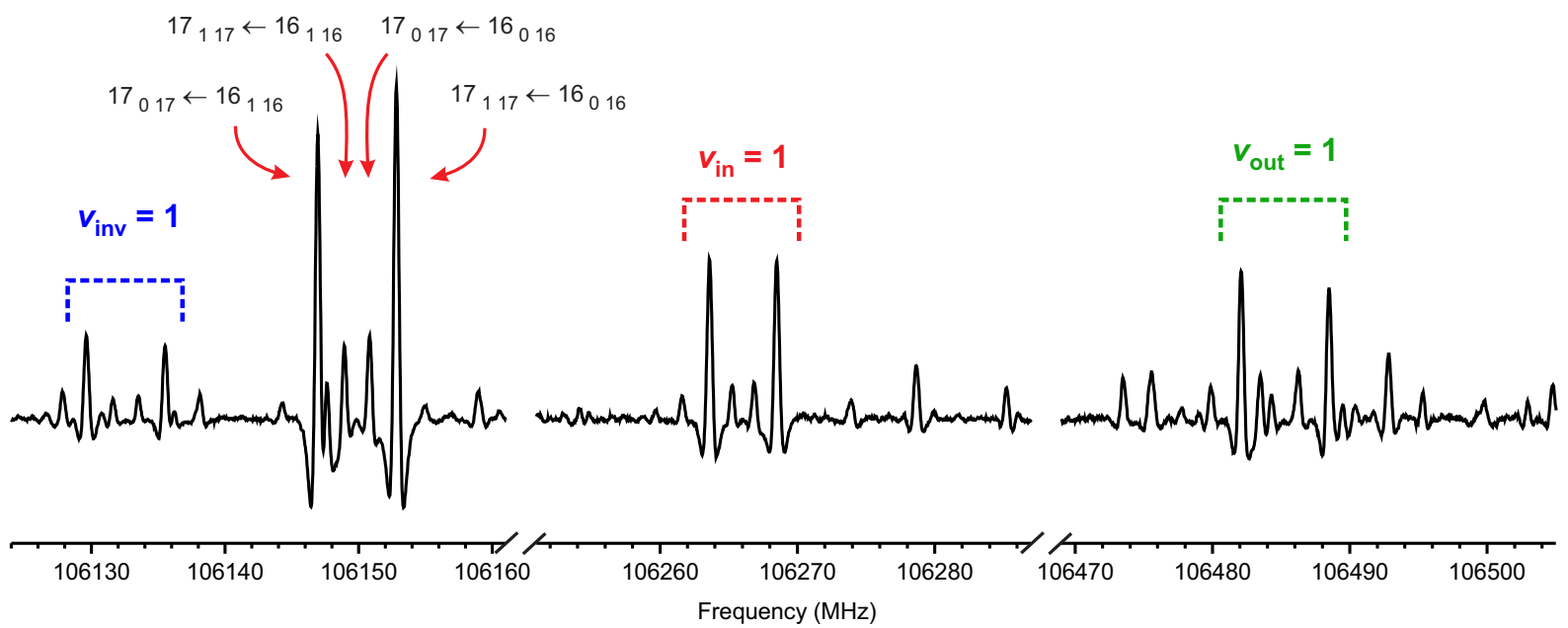

Fig. 4. Vibrational satellites accompanying the quartet of ground-state $J=17 \leftarrow 16$ transitions in the millimeter wave spectrum.

were identified and measured first. Then, we assigned $b$-type Qbranch transitions. As in the case of the ground state, several lines exhibited splitting due to ${ }^{14} \mathrm{~N}$ nuclear quadrupole coupling interactions. The above Hamiltonian $H=H_{\mathrm{R}}+H_{\mathrm{Q}}$ was therefore used to encompass all the assigned transitions. The obtained values of the rotational and centrifugal distortion constants as well as the ${ }^{14} \mathrm{~N}$ nuclear quadrupole coupling constants are collected in Table 1 . The list of the measured transition frequencies is given in Table 2.

Although the rotational transitions in $v_{\text {in }}=1, v_{\text {out }}=1$, and $v_{\text {inv }}=1$ states from Table 2 were amenable to single state fits, departures of the centrifugal distortion constant values can be noticed for $v_{\text {in }}=1$ and $v_{\text {out }}=1$ when compared to the groundstate values. It is worth noting that the average value of $\Delta_{K}$ for these two states $(-9.63 \mathrm{kHz})$ is very close to the ground-state value $(-10.058 \mathrm{kHz})$. The same behavior is also perceptible for other centrifugal distortion constants and points to the existence of interactions between $v_{\text {in }}=1$ and $v_{\text {out }}=1$. The spectroscopic constants reported in Table 1 should be thus taken as effective parameters that reproduce the vibrational satellite spectrum near the experimental uncertainty.

Since the spectroscopic line lists for interstellar detection require not only reliable line frequencies but also line intensities, we provide in Table 3 partition functions of propiolamide
Table 3. Partition functions of propiolamide.

\begin{tabular}{rrrr}
\hline \hline$T(\mathrm{~K})$ & $Q_{\text {rot }}$ & $Q_{\text {vib-rot }}{ }^{(a)}$ & $Q_{\text {vib }}$ \\
\hline 300.000 & 73387.03 & 149951.34 & 5.27 \\
225.000 & 47641.04 & 83083.57 & 2.73 \\
150.000 & 25920.14 & 35896.97 & 1.55 \\
75.000 & 9161.75 & 9704.56 & 1.06 \\
37.500 & 3240.31 & 3246.14 & 1.00 \\
18.750 & 1146.94 & 1146.94 & 1.00 \\
9.375 & 406.53 & 406.53 & 1.00 \\
\hline
\end{tabular}

Notes. ${ }^{(a)}$ This vibrational-rotational partition function includes the contribution of the ground state and $v_{\text {in }}=1, v_{\text {out }}=1$, and $v_{\text {inv }}=1$ excited vibrational states.

at multiple temperatures. The rotational partition function $\left(Q_{\mathrm{rot}}\right)$ corresponds to the ground vibrational state and was evaluated by summation over the energy levels. We used the SPCAT program (Pickett 1991) to undertake this summation numerically, employing the rotational and centrifugal distortion constants from Table 1, dipole moment components $\left|\mu_{a}\right|=1.08 \mathrm{D}$ and $\left|\mu_{b}\right|=3.51 \mathrm{D}$ from Little \& Gerry (1978), and the maximum value of the $J$ quantum number of 200 . The vibrational-rotational 


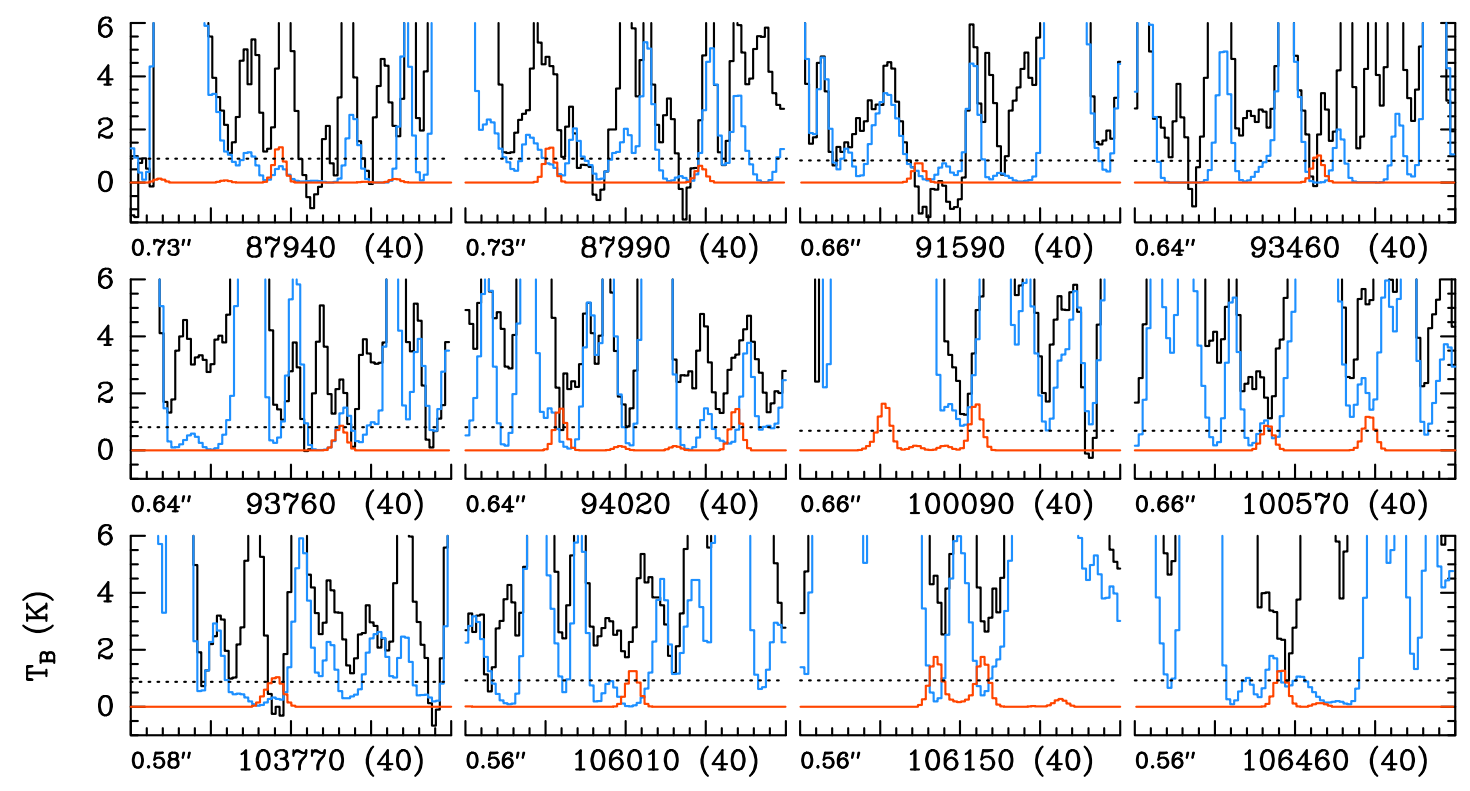

Fig. 5. Selection of transitions of $\mathrm{HCCC}(\mathrm{O}) \mathrm{NH}_{2}, v=0$ covered by our ALMA survey. The synthetic spectrum of $\mathrm{HCCC}(\mathrm{O}) \mathrm{NH}_{2}, v=0$ used to derive the upper limit to its column density is indicated in red and overlaid on the observed spectrum of Sgr B2(N1S) shown in black. The blue synthetic spectrum contains the contributions from all molecules identified in our survey so far, but not from the species shown in red. The central frequency and width are indicated in MHz below each panel. The angular resolution (HPBW) is also indicated. The $y$-axis is labeled in brightness temperature units $(\mathrm{K})$. The dotted line indicates the $3 \sigma$ noise level.

partition function $\left(Q_{\text {vib-rot }}\right)$ takes into consideration the ground state and the three observed excited vibrational states. This partition function was also computed by summation over the energy levels with the vibrational energies of 170,210 , and $300 \mathrm{~cm}^{-1}$ for $v_{\text {in }}=1, v_{\text {out }}=1$, and $v_{\text {inv }}=1$, respectively, and the rotational and centrifugal distortion constants from Table 1 . The values of the vibrational energies were roughly estimated by comparison of spectral intensities of the same rotational transitions in the corresponding excited vibrational state with respect to those in the ground state. Finally, the vibrational partition function $\left(Q_{\text {vib }}\right)$ was evaluated using Eq. (3.60) of Gordy \& Cook (1970) by taking into consideration the frequencies of 18 normal vibrational modes from Table A.1.

\section{Search for propiolamide toward Sgr B2(N)}

\subsection{Observations}

The data set used in this work was extracted from the ReMoCA survey performed with ALMA toward Sgr B2(N). Details about the observational setup and data reduction of this imaging spectral line survey can be found in Belloche et al. (2019). In short, the angular resolution (HPBW) of the survey varies between $\sim 0.3^{\prime \prime}$ and $\sim 0.8^{\prime \prime}$, with a median value of $0.6^{\prime \prime}$, which corresponds to $\sim 4900$ au at the distance of Sgr B2 $(8.2 \mathrm{kpc}$; Reid et al. 2019). The observations covered the frequency range from 84.1 to $114.4 \mathrm{GHz}$ with a spectral resolution of $488 \mathrm{kHz}(1.7$ to $1.3 \mathrm{~km} \mathrm{~s}^{-1}$ ). The equatorial coordinates of the phase center are $(\alpha, \delta)_{\mathrm{J} 2000}=\left(17^{\mathrm{h}} 47^{\mathrm{m}} 19^{\mathrm{s}} .87,-28^{\circ} 22^{\prime} 16^{\prime \prime}{ }^{\prime} 0\right)$. This position is located halfway between the two main hot molecular cores of Sgr B2(N) called Sgr B2(N1) and Sgr B2(N2), which are separated by $4.9^{\prime \prime}$ or $\sim 0.2 \mathrm{pc}$ in projection onto the plane of the sky. The sensitivity per spectral channel ranges between 0.35 and $1.1 \mathrm{mJy}$ beam $^{-1}(\mathrm{rms})$, with a median value of $0.8 \mathrm{mJy}^{\mathrm{beam}}{ }^{-1}$.

We selected two positions for this study: the offset position Sgr B2(N1S) located at $(\alpha, \delta)_{\mathrm{J} 2000}=\left(17^{\mathrm{h}} 47^{\mathrm{m}} 19^{\mathrm{s}} .870\right.$, $\left.-28^{\circ} 22^{\prime} 19^{\prime} 48\right)$ and the secondary hot core Sgr B2(N2) at $(\alpha, \delta)_{\mathrm{J} 2000}=\left(17^{\mathrm{h}} 47^{\mathrm{m}} 19^{\mathrm{s}} .863,-28^{\circ} 22^{\prime} 13^{\prime \prime} \cdot 27\right)$. Sgr B2(N1S) is about 1" to the south of the main hot core Sgr B2(N1) and was chosen by Belloche et al. (2019) for the lower opacity of its continuum emission, which allows for a deeper look into the molecular content of this hot core. We used a new version of our data set for which we have improved the splitting of the continuum and line emission as reported in Melosso et al. (2020).

The spectra of Sgr B2(N1S) and Sgr B2(N2) were modeled with the software Weeds (Maret et al. 2011) under the assumption of local thermodynamic equilibrium (LTE). This assumption is appropriate because the regions where hot-core emission is detected in Sgr B2(N) have high densities $\left(>1 \times 10^{7} \mathrm{~cm}^{-3}\right.$, see Bonfand et al. 2019). We derived a best-fit synthetic spectrum of each molecule separately and then added the contributions of all identified molecules together. We modeled each species with a set of five parameters: size of the emitting region $\left(\theta_{\mathrm{s}}\right)$, column density $(N)$, temperature $\left(T_{\text {rot }}\right)$, linewidth $(\Delta V)$, and velocity offset $\left(V_{\text {off }}\right)$ with respect to the assumed systemic velocity of the source, $V_{\text {sys }}=62 \mathrm{~km} \mathrm{~s}^{-1}$ for Sgr B2(N1S) and $V_{\text {sys }}=74 \mathrm{~km} \mathrm{~s}^{-1}$ for Sgr B2(N2).

\subsection{Nondetection of propiolamide}

We used the parameters derived for acetamide, $\mathrm{CH}_{3} \mathrm{C}(\mathrm{O}) \mathrm{NH}_{2}$, by Belloche et al. $(2017,2019)$ to compute LTE synthetic spectra of propiolamide and search for rotational emission of the latter in the ReMoCA spectra of Sgr B2(N2) and Sgr B2(N1S), respectively. We only kept the column density of propiolamide as a free parameter. We found no evidence for emission of propiolamide toward either source. The nondetection toward Sgr B2(N1S) and Sgr B2(N2) is illustrated in Figs. 5 and 6, respectively, and the upper limits to the column density of propiolamide are indicated in Tables 4 and 5, respectively. The tables also recall the parameters previously obtained for acetamide. The spectroscopic catalog used to compute the synthetic spectra of propiolamide 


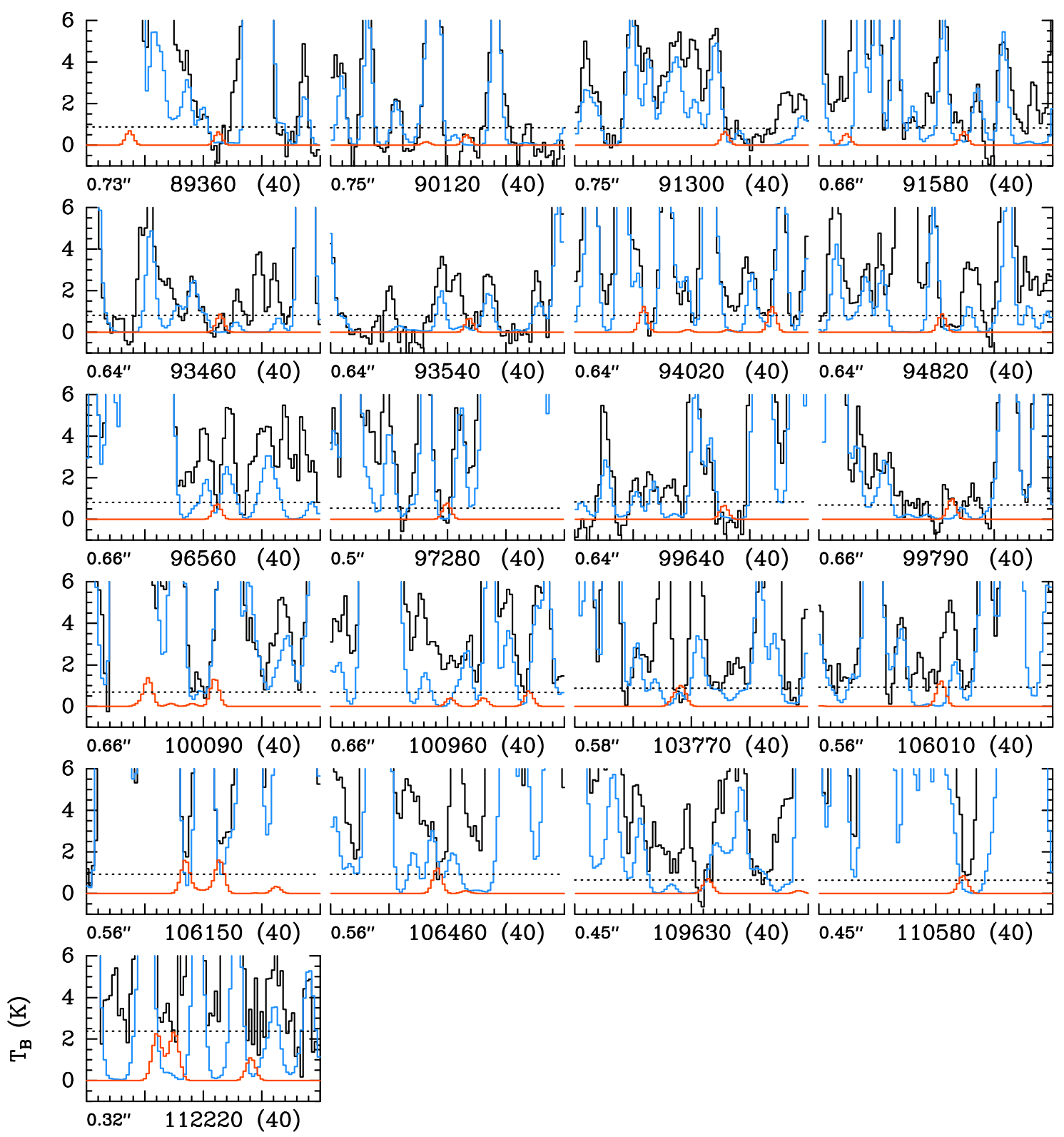

Fig. 6. Same as Fig. 5 but for Sgr B2(N2).

shown in Figs. 5 and 6 was prepared with the partition function that includes the vibrational ground state and the three lowest vibrationally excited states $\left(Q_{\text {vib-rot }}\right.$ in Table 3$)$. The correction factor $F_{\text {vib }}$ used in Tables 4 and 5 to derive the upper limit to the column density of propiolamide accounts for the higher vibrational states, using the corresponding vibrational frequencies listed in Table A.1. $F_{\text {vib }}$ was computed as $Q_{\text {vib }} \times Q_{\text {rot }} / Q_{\text {vib-rot }}$ using the values listed in Table 3.

\section{Discussion}

\subsection{Comparison to other molecules in $\operatorname{Sgr} B 2(N)$}

The nondetection of propiolamide toward Sgr B2(N1S) and Sgr B2(N2) reported in Sect. 4.2 implies that propiolamide is at least $\sim 50$ and $\sim 13$ times less abundant than acetamide toward Sgr B2(N1S) and Sgr B2(N2), respectively. For comparison, cyanoacetylene, $\mathrm{HC}_{3} \mathrm{~N}$, is about six times less abundant than methyl cyanide, $\mathrm{CH}_{3} \mathrm{CN}$, toward $\mathrm{Sgr}$ B2(N2) (Belloche et al. 2016) and a preliminary analysis of the ReMoCA survey yields a similar ratio for Sgr B2(N1S). Both pairs of molecules, the amides $\mathrm{HCCC}(\mathrm{O}) \mathrm{NH}_{2} / \mathrm{CH}_{3} \mathrm{C}(\mathrm{O}) \mathrm{NH}_{2}$ and the cyanides $\mathrm{HC}_{3} \mathrm{~N} / \mathrm{CH}_{3} \mathrm{CN}$, share the same structural difference (an unsaturated $\mathrm{C}_{2} \mathrm{H}$ group replaced with a saturated $\mathrm{CH}_{3}$ group), but the abundance difference between both (larger) amides is more pronunced than for the (smaller) cyanides, by at least a factor of 2 for Sgr B2(N2) and at least a factor of 8 for Sgr B2(N1S).

\subsection{Formation mechanisms for propiolamide}

Existing interstellar chemical networks do not include propiolamide; however, guided by the behavior of related species in the chemical kinetic simulations, we may speculate on the factors that could impact upon its formation and/or survival during star formation.

In common with acetamide, it would appear plausible that propiolamide could be produced on grain surfaces, or within their ice mantles, by the reaction of the radical $\mathrm{NH}_{2} \mathrm{CO}$ with another radical; $\mathrm{CH}_{3}$ in the former case, $\mathrm{C}_{2} \mathrm{H}$ in the latter. 
Table 4. Parameters of our best-fit LTE model of acetamide toward Sgr B2(N1S), and upper limit for propiolamide.

\begin{tabular}{|c|c|c|c|c|c|c|c|c|c|}
\hline Molecule & Status $^{(a)}$ & $N_{\text {det }}^{(b)}$ & $\begin{array}{c}\theta_{\mathrm{s}}^{(c)} \\
\left({ }^{\prime \prime}\right)\end{array}$ & $\begin{array}{c}T_{\text {rot }}{ }^{(d)} \\
(\mathrm{K})\end{array}$ & $\begin{array}{c}N^{(e)} \\
\left(\mathrm{cm}^{-2}\right)\end{array}$ & $F_{\mathrm{vib}}(f)$ & $\begin{array}{c}\Delta V^{(g)} \\
\left(\mathrm{km} \mathrm{s}^{-1}\right)\end{array}$ & $\begin{array}{c}V_{\text {off }^{(h)}} \\
\left(\mathrm{km} \mathrm{s}^{-1}\right)\end{array}$ & $\frac{N_{\mathrm{ref}}(i)}{N}$ \\
\hline $\mathrm{CH}_{3} \mathrm{C}(\mathrm{O}) \mathrm{NH}_{2}{ }^{(j)} \star$ & d & 153 & 2.0 & 160 & $4.1(17)$ & 1.16 & 5.0 & 0.0 & 1 \\
\hline $\mathrm{HCCC}(\mathrm{O}) \mathrm{NH}_{2}$ & $\mathrm{n}$ & 0 & 2.0 & 160 & $<8.5(15)$ & 1.18 & 5.0 & 0.0 & $>48$ \\
\hline
\end{tabular}

Notes. ${ }^{(a)}$ d: detection, n: nondetection. ${ }^{(b)}$ Number of detected lines (conservative estimate, see Sect. 3 of Belloche et al. 2016). One line of a given species may mean a group of transitions of that species that are blended together. ${ }^{(c)}$ Source diameter (FWHM). ${ }^{(d)}$ Rotational temperature. ${ }^{(e)}$ Total column density of the molecule. $x(y)$ means $x \times 10^{y} .{ }^{(f)}$ Correction factor that was applied to the column density to account for the contribution of vibrationally excited states, in the cases where this contribution was not included in the partition function of the spectroscopic predictions. ${ }^{(g)}$ Linewidth (FWHM). ${ }^{\left({ }^{h}\right)}$ Velocity offset with respect to the assumed systemic velocity of Sgr B2(N1S), $V_{\text {sys }}=62 \mathrm{~km} \mathrm{~s}^{-1}$. ${ }^{(i)}$ Column density ratio, with $N_{\text {ref }}$ the column density of the previous reference species marked with a $\star .{ }^{(j)}$ The parameters were derived from the ReMoCA survey by Belloche et al. (2019).

Table 5. Parameters of our best-fit LTE model of acetamide toward Sgr B2(N2), and upper limit for propiolamide.

\begin{tabular}{|c|c|c|c|c|c|c|c|c|c|}
\hline Molecule & $\operatorname{Status}^{(a)}$ & $N_{\text {det }}{ }^{(b)}$ & $\begin{array}{c}\theta_{\mathrm{s}}(c) \\
\left({ }^{\prime \prime}\right) \\
\end{array}$ & $\begin{array}{c}T_{\text {rot }}(d) \\
(\mathrm{K}) \\
\end{array}$ & $\begin{array}{c}N^{(e)} \\
\left(\mathrm{cm}^{-2}\right)\end{array}$ & $F_{\text {vib }}(f)$ & $\begin{array}{c}\Delta V^{(g)} \\
\left(\mathrm{km} \mathrm{s}^{-1}\right)\end{array}$ & $\begin{array}{c}V_{\text {off }}{ }^{(h)} \\
\left(\mathrm{km} \mathrm{s}^{-1}\right)\end{array}$ & $\frac{N_{\text {ref }}}{N}(i)$ \\
\hline $\mathrm{CH}_{3} \mathrm{C}(\mathrm{O}) \mathrm{NH}_{2}{ }^{(j) \star}$ & d & 23 & 0.9 & 180 & 1.4 (17) & 1.23 & 5.0 & 1.5 & 1 \\
\hline $\mathrm{HCCC}(\mathrm{O}) \mathrm{NH}_{2}$ & $\mathrm{n}$ & 0 & 0.9 & 180 & $<1.0(16)$ & 1.30 & 5.0 & 0.0 & $>13$ \\
\hline
\end{tabular}

Notes. ${ }^{(a)}$ d: detection, n: nondetection. ${ }^{(b)}$ Number of detected lines (conservative estimate, see Sect. 3 of Belloche et al. 2016). One line of a given species may mean a group of transitions of that species that are blended together. ${ }^{(c)}$ Source diameter (FWHM). ${ }^{(d)}$ Rotational temperature. ${ }^{(e)}$ Total column density of the molecule. $x(y)$ means $x \times 10^{y} .{ }^{(f)}$ Correction factor that was applied to the column density to account for the contribution of vibrationally excited states, in the cases where this contribution was not included in the partition function of the spectroscopic predictions. ${ }^{(g)}$ Linewidth (FWHM). ${ }^{(h)}$ Velocity offset with respect to the assumed systemic velocity of Sgr B2(N2), $V_{\text {sys }}=74 \mathrm{~km} \mathrm{~s}^{-1}$. ${ }^{(i)}$ Column density ratio, with $N_{\text {ref }}$ the column density of the previous reference species marked with a $\star .{ }^{(j)}$ The parameters were derived from the EMoCA survey by Belloche et al. (2017).

Unsaturated hydrocarbons such as acetylene $\left(\mathrm{C}_{2} \mathrm{H}_{2}\right)$ and associated radicals such as $\mathrm{C}_{2} \mathrm{H}$, are typically considered "early-time" species, which become abundant in the gas phase before free carbon has had time to become locked up in stable species such as CO. At such a stage, the dust-grain ice mantles are still in the process of formation. Surface radical reactions during this period might provide an opportunity for propiolamide production, while gas-phase hydrocarbons remain available to adsorb onto the grain surfaces. Although the grains would be considered too cold $(\sim 10 \mathrm{~K})$ for surface diffusion of large radicals to drive production, the occasional presence of reactive radicals in close proximity to each other appears to be sufficient to allow a degree of complex organic molecule production even at low temperatures (e.g., Jin \& Garrod 2020). New models of hot-core chemistry (Garrod et al., in prep.) indicate that such cold, early mechanisms may be major contributors to the production of certain species that are later observed in the gas phase. This appears to be the case with acetamide; if similar cold mechanisms were also to contribute to propiolamide production, then the ratio of methane $\left(\mathrm{CH}_{4}\right)$ to acetylene produced in the models could give an indication of the relative production of the larger species. At the end of the cold collapse stage, the Garrod et al. (in prep.) models indicate that methane is around 3 orders of magnitude more abundant than acetylene in the ices. This ratio is consistent with the observational ratio of acetamide to the upper limit for propiolamide.

In the models, acetamide may also be formed on the grains through the addition of the radicals $\mathrm{NH}_{2}$ and $\mathrm{CH}_{3} \mathrm{CO}$, the latter of which is a product of the destruction of acetaldehyde $\left(\mathrm{CH}_{3} \mathrm{CHO}\right)$. The comparable mechanism for propiolamide production would be $\mathrm{NH}_{2}$ addition to the $\mathrm{HC}_{2} \mathrm{CO}$ radical, which could be formed by the loss of a hydrogen atom from the aldehyde group of propynal, $\mathrm{HC}_{2} \mathrm{CHO}$. As this molecule and its surrounding chemistry are not included in our chemical models (other than in the most trivial fashion), the implications of such a mechanism cannot be quantified.

An alternative to cold surface production at early times is the cosmic-ray-induced UV photolysis of molecules within the dust-grain ice mantles, which may occur continuously from the onset of mantle formation until their ultimate desorption into the gas phase. This mechanism provides a means by which the requisite radicals can be produced and thence react to form propiolamide and acetamide. In this case, the relative abundances of precursors $\mathrm{NH}_{2} \mathrm{CHO}, \mathrm{CH}_{4}$ (and/or $\mathrm{CH}_{3} \mathrm{OH}$ ) and $\mathrm{C}_{2} \mathrm{H}_{2}$, as well as $\mathrm{NH}_{3}, \mathrm{CH}_{3} \mathrm{CHO}$ and $\mathrm{HC}_{2} \mathrm{CHO}$, would again be important in determining ratios between acetamide and propiolamide.

Assuming that propiolamide may be formed in some abundance on dust grains prior to the thermal desorption of dust-grain ices, it is also possible that reactions with abundant grainsurface $\mathrm{H}$ atoms could produce at least partial conversion to propanamide $\left(\mathrm{CH}_{3} \mathrm{CH}_{2} \mathrm{CONH}_{2}\right)$, further reducing the abundance of propiolamide. Hydrogenation by $\mathrm{H}$ atoms could similarly act to reduce the abundance of acetylene in the ice mantles, removing a possible precursor for the ongoing production of propiolamide via ice photolysis. All in all, the observed abundances and ratios between propiolamide and acetamide appear to be consistent with the expectations from limited chemical modeling evidence.

\section{Conclusions}

Using a combination of time-domain FTMW spectroscopy and frequency-domain millimeter wave spectroscopy techniques, a detailed rotational study of propiolamide from 6 to $440 \mathrm{GHz}$ was 
carried out. In total, more than 5500 new rotational lines for the ground state and the three lowest excited vibrational states were measured and assigned. The present work significantly extends the frequency coverage of the propiolamide rotational spectrum known to date and newly derived spectroscopic parameters provide a firm base to guide a search for this molecule using radio astronomy.

Propiolamide was not detected toward the hot molecular cores Sgr B2(N1S) and Sgr B2(N2) with ALMA. The upper limits derived for its column density imply that it is at least 50 and 13 times less abundant than acetamide toward these sources, respectively. This abundance difference between both amides is more pronounced than for their corresponding nitriles by at least a factor of 8 for Sgr B2(N1S) and 2 for Sgr B2(N2).

The observational results seem consistent with the low ratio of propiolamide to acetamide inferred from the results of chemical kinetic models (which include the latter species but not the former). In the proposed scenario, production of propiolamide would occur through radical addition on dust-grain surfaces. This mechanism may be most effective at early times in the chemical evolution when unsaturated hydrocarbons should be abundant in the gas phase. Reactions with grain-surface $\mathrm{H}$ atoms could also diminish the abundance of propiolamide and its precursors in the ice mantles prior to thermal desorption.

More promising sources for the detection of propiolamide may be sources where unsaturated carbon chain molecules are more prominent than in Sgr B2(N). One possibility, for instance, is $\mathrm{TMC} 1$, where $\mathrm{HC}_{3} \mathrm{~N}$ is more than one order of magnitude more abundant than $\mathrm{CH}_{3} \mathrm{CN}$ (Gratier et al. 2016). However, the amine $\mathrm{CH}_{3} \mathrm{NH}_{2}$ has not been detected toward TMC1 in the GOTHAM survey with the GBT telescope so far (B. McGuire, priv. comm. 2021), which is probably not a good sign for propiolamide. The quiescent source G+0.693-0.027, which is located in the vicinity of Sgr B2(N) and where propargylimine, HCC$\mathrm{CHNH}$, was recently detected by Bizzocchi et al. (2020), may be a more promising source to continue the search for propiolamide.

Acknowledgements. The funding from Ministerio de Ciencia e Innovación (CTQ2016-76393-P and PID2019-111396GB-I00), Czech Science Foundation (GACR, grant 19-25116Y), Junta de Castilla y León (Grants VA077U16 and VA244P20), and European Research Council under the European Union's Seventh Framework Programme ERC-2013-SyG, Grant Agreement n. 610256 NANOCOSMOS are gratefully acknowledged. E.R.A. thanks Fundación Biofísica Bizkaia (FBB) for a postdoctoral contract. J.C.G. thanks the program Physique et Chimie du Milieu Interstellaire (INSU-CNRS) and the Centre National d'Etudes Spatiales (CNES). This paper makes use of the following ALMA data: ADS/JAO.ALMA\#2016.1.00074.S. ALMA is a partnership of ESO (representing its member states), NSF (USA), and NINS (Japan), together with NRC (Canada), NSC and ASIAA (Taiwan), and KASI (Republic of Korea), in cooperation with the Republic of Chile. The Joint ALMA Observatory is operated by ESO, AUI/NRAO, and NAOJ. The interferometric data are available in the ALMA archive at https://almascience.eso.org/aq/. Part of this work has been carried out within the Collaborative Research Centre 956, subproject B3, funded by the Deutsche Forschungsgemeinschaft (DFG) - project ID 184018867. R.T.G. acknowledges support from the National Science Foundation (grant No. AST 19-06489).

\section{References}

Alonso, E. R., Kolesniková, L., Peña, I., et al. 2015, J. Mol. Spectr., 316, 84 Alonso, E. R., Kolesniková, L., Białkowska-Jaworska, E., et al. 2018, ApJ, 861, 70

Belloche, A., Menten, K. M., Comito, C., et al. 2008, A\&A, 482, 179

Belloche, A., Müller, H. S. P., Garrod, R. T., \& Menten, K. M. 2016, A\&A, 587 A91

Belloche, A., Meshcheryakov, A. A., Garrod, R. T., et al. 2017, A\&A, 601, A49

Belloche, A., Garrod, R. T., Müller, H. S. P., et al. 2019, A\&A, 628, A10

Bizzocchi, L., Prudenzano, D., Rivilla, V. M., et al. 2020, A\&A, 640, A98

Bonfand, M., Belloche, A., Garrod, R. T., et al. 2019, A\&A, 628, A27
Frisch, M. J., Trucks, G. W., Schlegel, H. B., et al. 2016, Gaussian16 Revision B.01 (Wallingford CT: Gaussian Inc.)

Fuchs, G. W., Cuppen, H. M., Ioppolo, S., et al. 2009, A\&A, 505, 629

Gordy, W., \& Cook, R. L. 1970, Microwave Molecular Spectra (Hoboken: Interscience Publishers)

Gordy, W., \& Cook, R. L. 1984, Microwave Molecular Spectra (New York: Wiley Interscience)

Gratier, P., Majumdar, L., Ohishi, M., et al. 2016, ApJS, 225, 25

Herbst, E., \& van Dishoeck, E. F. 2009, ARA\&A, 47, 427

Hiraoka, K., Ohashi, N., Kihara, Y., et al. 1994, Chem. Phys. Lett., 229, 408

Jin, M., \& Garrod, R. T. 2020, ApJS, 249, 26

Jonusas, M., Guillemin, J.-C., \& Krim, L. 2017, MNRAS, 468, 4592

Kisiel, Z., \& Pszczolkowski, L. 1994, J. Mol. Spectr., 166, 32

Kisiel, Z., Pszczółkowski, L., Medvedev, I. R., et al. 2005, J. Mol. Spectr., 233, 231

Kisiel, Z., Pszczółkowski, L., Drouin, B. J., et al. 2012, J. Mol. Spectr., 280, 134 Kolesniková, L., Alonso, E. R., Mata, S., \& Alonso, J. L. 2017a, ApJS, 229, 26 Kolesniková, L., Alonso, E. R., Mata, S., Cernicharo, J., \& Alonso, J. L. 2017b, ApJS, 233, 24

Kolesniková, L., Daly, A., Alonso, J., Tercero, B., \& Cernicharo, J. 2013, J. Mol. Spectr., 289, 13

Krim, L., Guillemin, J.-C., \& Woon, D. E. 2019, MNRAS, 485, 5210

León, I., Alonso, E. R., Mata, S., et al. 2017, Phys. Chem. Chem. Phys., 19, 24985

Little, G. B., \& Gerry, M. 1978, J. Mol. Spectr., 71, 321

Maret, S., Hily-Blant, P., Pety, J., Bardeau, S., \& Reynier, E. 2011, A\&A, 526, A47

Marstokk, K.-M., Møllendal, H., \& Samdal, S. 1996, J. Mol. Struct., 376, 11

Marstokk, K.-M., Møllendal, H., \& Samdal, S. 2000, J. Mol. Struct., 524, 69

McGuire, B. A. 2018, ApJS, 239, 17

Melosso, M., Belloche, A., Martin-Drumel, M.-A., et al. 2020, A\&A, 641, A160

Miller, F. A., \& Lemmon, D. H. 1967, Spectrochim. Acta A, 23, 1415

Pickett, H. M. 1991, J. Mol. Spectr., 148, 371

Pirim, C., \& Krim, L. 2011, Phys. Chem. Chem. Phys., 13, 19454

Reid, M. J., Menten, K. M., Brunthaler, A., et al. 2019, ApJ, 885, 131

Sanz-Novo, M., Belloche, A., Alonso, J. L., et al. 2020, A\&A, 639, A135

Theule, P., Borget, F., Mispelaer, F., et al. 2011, A\&A, 534, A64

Watanabe, N., \& Kouchi, A. 2002, ApJ, 571, L173

Watson, J. K. G. 1977, in Vibrational Spectra and Structure, ed. J. R. Durig (Amsterdam: Elsevier), 6, 1

\section{Appendix A: Complementary table}

Table A.1. Frequencies of normal vibrational modes of propiolamide used to calculate the vibrational partition function.

\begin{tabular}{rrr}
\hline \hline Mode & Frequency $\left(\mathrm{cm}^{-1}\right)$ & Symmetry \\
\hline 1 & 3724 & $A^{\prime}$ \\
2 & 3588 & $A^{\prime}$ \\
3 & 3471 & $A^{\prime}$ \\
4 & 2209 & $A^{\prime}$ \\
5 & 1747 & $A^{\prime}$ \\
6 & 1614 & $A^{\prime}$ \\
7 & 1329 & $A^{\prime}$ \\
8 & 1090 & $A^{\prime}$ \\
9 & 797 & $A^{\prime}$ \\
10 & 701 & $A^{\prime}$ \\
11 & 600 & $A^{\prime}$ \\
12 & 490 & $A^{\prime}$ \\
13 & 170 & $A^{\prime}$ \\
14 & 762 & $A^{\prime \prime}$ \\
15 & 723 & $A^{\prime \prime}$ \\
16 & 554 & $A^{\prime \prime}$ \\
17 & 300 & $A^{\prime \prime}$ \\
18 & 230 & $A^{\prime \prime}$ \\
\hline
\end{tabular}

Notes. The frequencies of the three lowest modes were estimated on the basis of the experimental relative intensities measurements with uncertainties of $30 \mathrm{~cm}^{-1}$, while the others were taken from the theoretical calculations at the B3LYP/6-311++G(d,p) level of the theory (Gaussian 16 package, Frisch et al. 2016). 